\title{
Nicotinamide N-methyltransferase inhibits autophagy induced by oxidative stress through suppressing the AMPK pathway in breast cancer cells
}

Haitao Yu ${ }^{1,2+}$, Xi Zhou ${ }^{1,2+}$, Yanzhong Wang ${ }^{1,2,3}$, Xucheng Huang ${ }^{1,2}$, Jun Yang ${ }^{1,2}$, Jin Zeng ${ }^{1,2}$, Guoli Li, ${ }^{1,2}$, Xinyou $\mathrm{Xie}^{1,2,3}$ and Jun Zhang ${ }^{1,2,3^{*}}$

\begin{abstract}
Background: Nicotinamide N-methyltransferase (NNMT) is highly expressed in several cancers and can regulate cell epigenetic status and various cell metabolism pathways, such as ATP synthesis and cellular stress response. We reported in our previous papers that NNMT overexpression inhibits the apoptosis and enhances the chemotherapy resistance of breast cancer cells. This study aims to investigate the effect of NNMT on autophagy induced by oxidative stress in breast cancer cells, which might provide a novel therapeutic strategy for breast cancer treatment.

Methods: NNMT and LC3B II protein levels in the two cell models (SK-BR-3 and MDA-MB-231) with NNMT overexpression or knockdown were detected by Western blotting and correlated with each other. Changes in cellular viability, intracellular reactive oxygen species (ROS) and ATP levels were assessed after $\mathrm{H}_{2} \mathrm{O}_{2}$ treatment. Then, autophagosomes were imaged by transmission electron microscopy, and LC3 puncta were examined by confocal microscopy and flow cytometry. The LC3B II level and AMPK-ULK1 pathway activity were both detected by Western blotting to determine the role of NNMT in the $\mathrm{H}_{2} \mathrm{O}_{2}$-induced autophagy.

Results: NNMT expression was negatively correlated with LC3B II expression in both cell models (SK-BR-3 and MDAMB-231). Then, NNMT overexpression attenuated the autophagy induced by $\mathrm{H}_{2} \mathrm{O}_{2}$ in SK-BR-3 cells, whereas knockdown promoted autophagy induced by $\mathrm{H}_{2} \mathrm{O}_{2}$ in MDA-MB-231 cells. Furthermore, mechanistic studies showed that NNMT suppressed the ROS increase, ATP decrease and AMPK-ULK1 pathway activation, resulting in the inhibition of $\mathrm{H}_{2} \mathrm{O}_{2}$-induced autophagy in breast cancer cells.

Conclusions: We conclude that NNMT inhibits the autophagy induced by oxidative stress through the ROS-mediated AMPK-ULK1 pathway in breast cancer cells and may protect breast cancer cells against oxidative stress through autophagy suppression.
\end{abstract}

Keywords: Nicotinamide N-methyltransferase, Autophagy, Oxidative stress, AMPK, ULK1, Breast cancer

*Correspondence: jameszhang2000@zju.edu.cn

${ }^{\dagger}$ Haitao Yu, Xi Zhou have contributed equally to this work

${ }^{1}$ Department of Clinical Laboratory, Sir Run Run Shaw Hospital, Zhejiang University School of Medicine, 3 East Qingchun Road, Hangzhou 310016, Zhejiang, People's Republic of China

Full list of author information is available at the end of the article

\section{Background}

Autophagy is a highly conserved catabolic biological process that enables cells to degrade damaged or unwanted proteins and organelles in lysosomes; thus, it plays a critical role in the recycling of intracellular components and the quality control of proteins and organelles to protect

c) The Author(s) 2020. This article is licensed under a Creative Commons Attribution 4.0 International License, which permits use, sharing, adaptation, distribution and reproduction in any medium or format, as long as you give appropriate credit to the original author(s) and the source, provide a link to the Creative Commons licence, and indicate if changes were made. The images or other third party material in this article are included in the article's Creative Commons licence, unless indicated otherwise in a credit line to the material. If material is not included in the article's Creative Commons licence and your intended use is not permitted by statutory regulation or exceeds the permitted use, you will need to obtain permission directly from the copyright holder. To view a copy of this licence, visit http://creativecommons.org/licenses/by/4.0/. The Creative Commons Public Domain Dedication waiver (http://creativecommons.org/publicdomain/zero/1.0/) applies to the data made available in this article, unless otherwise stated in a credit line to the data. 
intracellular homeostasis [1,2]. Although a basal level of autophagy is generally occurs under physiological conditions as part of a cellular repair process, it can be strongly activated in pathological conditions by various stress stimuli, including nutrient starvation and oxidative stress [3], leading to distinct cell fate. Emerging evidence shows that dysfunction of autophagy may lead to a number of diseases, such as metabolic disease and cancer. In cancer progression, autophagy is generally a double-edged sword and its exact role in cancer depends on tumour type, stage, and so on [4]. Recently, much evidence has revealed that the induction or suppression of autophagy can impact cancer status, thus modulating autophagy activity by targeting autophagy regulatory molecules may be a new autophagy-based therapeutic intervention for human cancer treatment [5].

Nicotinamide N-methyltransferase (NNMT), a phase II metabolizing enzyme, mainly transfers a methyl group from S-adenosyl-L-methionine (SAM) to nicotinamide (NAM), producing 1-methylnicotinamide (1MNA) and $\mathrm{S}$-adenosylhomocysteine (SAH). Therefore, NNMT participates in the intracellular methylation cycle, which affects the global methylation status and metabolome of cells [6]. In the past decade, NNMT was found to be highly expressed in many kinds of tumour [7-11] and was found to alter various cancer cell metabolism pathways to regulate the cellular stress response $[12,13]$ and epigenetic state, which results in high expression of protumour genes [14]. In our previous study, we found that NNMT and its product 1MNA can decrease the mitochondria-mediated apoptosis by suppressing intracellular ROS in breast cancer cells [15]. Recently, we reported that NNMT is overexpressed in breast cancer patients' tumours and increases the resistance to chemotherapy via its product 1MNA. However, its effect on autophagy regulation in breast cancer has not yet been investigated.

In this study, we examined the expression of NNMT and LC3B II, a marker of autophagy in breast cancer cell line models with NNMT overexpression or knockdown, and then determined correlation between them. Next, we utilized $\mathrm{H}_{2} \mathrm{O}_{2}$ to induce autophagy and detected the levels of autophagosomes, LC3 puncta and LC3B II in cell line models to determine the role of NNMT expression in autophagy regulation. In addition, cell activity, ROS, ATP and autophagy related signalling pathways were also detected to further discover NNMT's regulation of autophagy induced by $\mathrm{H}_{2} \mathrm{O}_{2}$.

\section{Methods}

Antibodies

The primary antibodies that included anti-LC3 (\#12741), anti-p-AMPK (T172) (\# 2535), anti-AMPK (\#2532), anti-p-ULK1 (Ser317) (\# 12753), anti-ULK1 (\# 6439), anti- $\beta$-Actin (\# 4970) and goat anti-rabbit (\# 7074) and goat anti-mouse (\# 7076) HRP-conjugated secondary antibodies were all obtained from Cell Signaling Technology (Beverly, Massachusetts, USA). The monoclonal antibody of NNMT was prepared in our lab as previously described [15]. The $\mathrm{H}_{2} \mathrm{O}_{2}$ solution was obtained from Sigma (\#H1009).

\section{Cell lines and cell culture}

The human breast cancer cell lines MDA-MB-231, MDA-MB-468, BT549, MCF7 and SK-BR-3 were purchased from American Type Culture Collection (ATCC, USA). Cells were cultured in Dulbecco's modified Eagle medium (Gibco, USA) containing 10\% foetal bovine serum (Gibco, USA) and $100 \mu \mathrm{g} / \mathrm{ml}$ penicillin-streptomycin (Sigma, USA) in a humidified incubator supplemented with $5 \% \mathrm{CO}_{2}$ at $37^{\circ} \mathrm{C}$.

\section{Lentiviral vectors and infection}

The lentivirus with Plenti-Pur-NNMT or pGCSIL-PurshRNA-NNMT vector was purchased from GeneChem Co., Ltd. (Shanghai, China). The lentivirus with the Plenti-Pur-NNMT vector was infected into SK-BR-3 cells to overexpress NNMT, and the lentivirus with the pGCSIL-PUR-shRNA-NNMT vector was infected into MDA-MB-231 cells to silence NNMT. Plenti-Pur and pGCSIL-Pur-shRNA-NC were used as controls. After infection, cells were selected with puromycin $(1 \mu \mathrm{M})$ for 5 days.

\section{Western blot analysis}

Cell proteins were extracted by lysis buffer (Cell Signaling, USA) with a complete protease inhibitor cocktail (Sigma, USA) on ice. Protein concentrations were quantified using a BCA protein assay kit (Beyotime, China). Eighty micrograms of protein sample was mixed with $5 \times$ loading buffer and loaded into the lanes of $10 \%$ sodium dodecyl sulfate-polyacrylamide gel (SDS). The proteins were separated by electrophoresis and transferred to $0.2 \mu \mathrm{M}$ PVDF membranes (Millipore, USA). After blocking with $5 \%$ nonfat milk (Sigma, USA) for $2 \mathrm{~h}$ and washing with TBS-T three times at room temperature, the membranes were incubated with primary antibodies at $4{ }^{\circ} \mathrm{C}$ overnight. After washing with TBS-T for three times, the membranes were incubated with appropriate HRP-conjugated secondary antibodies for $1 \mathrm{~h}$ at room temperature. Protein bands were detected using an ECL detection reagent (Millipore, USA) and imaged by the Image Quant LAS-4000 instrument (Fujifilm, Japan). The band density was analysed by ImageJ and normalized to the level of $\beta$-actin in each group. The experiments were repeated independently for three times. 


\section{Cell viability by MTS assay}

Cell viability was determined by the MTS assay. The $200 \mu \mathrm{l}$ cells were seeded into 96-well flat-bottom plates with 8000 cells per well. After incubation for overnight at $37{ }^{\circ} \mathrm{C}$ and $5 \% \mathrm{CO} 2$, cells were treated with $\mathrm{H}_{2} \mathrm{O}_{2}(100$ or $200 \mu \mathrm{M})$ or $\mathrm{H}_{2} \mathrm{O}$ as a control for $8 \mathrm{~h}$. Then, $20 \mu \mathrm{l}$ MTS reagent (Promega, USA) was added into each well and cells were incubated for another $3 \mathrm{~h}$. Finally, the absorbance value of each well was read at $490 \mathrm{~nm}$ using a microplate reader instrument (BIO RAD, Model 680, Japan) and normalized to that of the control group, which was defined as 1 . All experiments were performed independently three times using five wells for each group.

\section{Measurement of cellular reactive oxygen species by flow cytometry}

Reactive oxygen species (ROS) were measured by flow assay. Cells were added to 6-well plates and treated with $\mathrm{H}_{2} \mathrm{O}_{2}(100$ or $200 \mu \mathrm{M})$ or $\mathrm{H}_{2} \mathrm{O}$ as a control for $8 \mathrm{~h}$ after culture overnight. Then, the cells were washed in HBSS, collected and incubated in $1 \mathrm{~mL}$ staining buffer with $10 \mu \mathrm{l}$ H2DCF-DA (10 mM) (Sigma, USA) for $30 \mathrm{~min}$ at $37{ }^{\circ} \mathrm{C}$. Finally, the cells were resuspended in $0.5 \mathrm{~mL}$ PBS with $2 \%$ FBS after separation from the staining buffer. The fluorescence intensity of H2DCF-DA was analysed by a FACSCalibur flow cytometer (BD, USA) at $485 \mathrm{~nm}$ excitation and $530 \mathrm{~nm}$ emission to indicate the level of ROS in cells. The data were analysed using Flow Jo 7.5.5 software. The mean fluorescence intensity was normalized to that of the control group, which was defined as 1 . The experiment was conducted independently three times.

\section{Measurement of cellular ATP}

The intracellular ATP was measured using the CellTiterGlo 2.0 reagent kit (Promega, \#G9242, USA). Briefly, cells were seeded into the a 96-well clear flat bottom plate (Corning, \#3596, USA) with 4000 cells per well. After culturing for $24 \mathrm{~h}$, cells were treated with $\mathrm{H}_{2} \mathrm{O}_{2}$ (100 or $200 \mu \mathrm{M})$ or $\mathrm{H}_{2} \mathrm{O}$ as a control for $8 \mathrm{~h}$. Then, $100 \mu \mathrm{l} \mathrm{CellTi-}$ ter-Glo 2.0 reagent was added into each well. To lyse cells and stabilize the luminescent signal, the plate was mixed on an orbital shaker for $2 \mathrm{~min}$ and incubated at room temperature for $10 \mathrm{~min}$. Finally, the value of luminescence in each well was recorded by a luminometer (Turner Designs, TD-20/20, USA) to indicate the ATP level. The mean ATP level was normalized to that of the control group, which was defined as 1 . The experiment was performed independently three times.

\section{Transmission electron microscopy (TEM)}

The autophagic vacuoles were examined by transmission electron microscopy. Following $\mathrm{H}_{2} \mathrm{O}_{2}(200 \mu \mathrm{M})$ treatment or $\mathrm{H}_{2} \mathrm{O}$ as a control for $8 \mathrm{~h}$, cells were fixed with $2.5 \%$ phosphate-buffered glutaraldehyde overnight at $4{ }^{\circ} \mathrm{C}$. The next day, cells were post-fixed with $1 \%$ osmium tetroxide $\left(\mathrm{OsO}_{4}\right)$ for $1 \mathrm{~h}$ after being washed with PBS three times for $15 \mathrm{~min}$. Then, the cells were washed with $\mathrm{ddH}_{2} \mathrm{O}$ and stained with $2 \%$ uranyl acetate for $30 \mathrm{~min}$ at room temperature. After that, cells were dehydrated with an increasing gradient of ethanol (50\%, 70\%, and 90\%) for 15 min each, ethanol (100\%) for $20 \mathrm{~min}$, and $100 \%$ acetone for $20 \mathrm{~min}$ and embedded into epoxy resin. A 60-80 nm ultrathin section was prepared from each sample by a diamond knife on a Leica Ultracut UCT (Wetzlar, Leica Microsystems $\mathrm{GmbH}$, Germany) and adhered to uncoated 200-mesh copper grids. Then, each sample was stained with $2 \%$ uranyl acetate and lead citrate for 15 min each. Finally, each sample was observed by TEM (Tokyo, JEM-1400/ JEM-1400 PLUS, Japan) at $80 \mathrm{kV}$. Autophagic vacuoles were imaged by the transmission electron microscopy (Tokyo, Hitachi H-7000FA, Japan). Images of five view fields in each group were randomly taken for data analysis. The average number of autophagic vacuoles per cell was recorded. The experiment was performed independently three times.

\section{LC3 puncta formation assay by confocal microscopy and flow cytometry}

To analyse autophagic flux, cells were seeded in a 4-well chamber slider (LAB TEK, Rochester, NY) at a density of $2 \times 10^{4}$ cells/well and transfected with a pEGFP-LC3 plasmid (Addgene \#24920). Following $\mathrm{H}_{2} \mathrm{O}_{2}(200 \mu \mathrm{M})$ treatment or $\mathrm{H}_{2} \mathrm{O}$ as a control for $8 \mathrm{~h}$, autophagy was observed under a laser scanning confocal microscope (Olympus). Five representative fields were captured at $200 \times$ magnification, and then the number of LC3 puncta in cells was recorded. The fluorescence intensity of GFP-LC3 in cells after treatment was also analysed by flow cytometry. The mean of fluorescence intensity was normalized to that of the control group, which was defined as 1 . The two experiments were both performed independently three times.

\section{Statistical analysis}

All data are presented as the mean \pm SEM. The statistical analyses were performed using the SPSS 22.0 statistical software package. Differences between two groups with different treatments were analysed using two-tailed independent-samples Student's t-test. Statistical significance was defined as $* p<0.05,{ }^{* *} p<0.01$, **** $p<0.001$ and $* * * * 0<0.0001$. 


\section{Results}

NNMT reduces autophagy in breast cancer cells

To determine the effect of NNMT on regulating autophagy in breast cancer cells, we examined the protein level of NNMT in the five cell lines. Among these cell lines, SK-BR-3, MCF7 and MDA-MB-468 showed either no or low NNMT expression, and BT549 showed a medium expression level, while MDA-MB-231 showed the highest level (Fig. 1a, b). Therefore, the SK-BR-3 cell line with no NNMT expression was infected with the lentivirus of Plenti-Pur-NNMT to overexpress NNMT or Plenti-Pur as control, whereas the MDA-MB-231 cell line
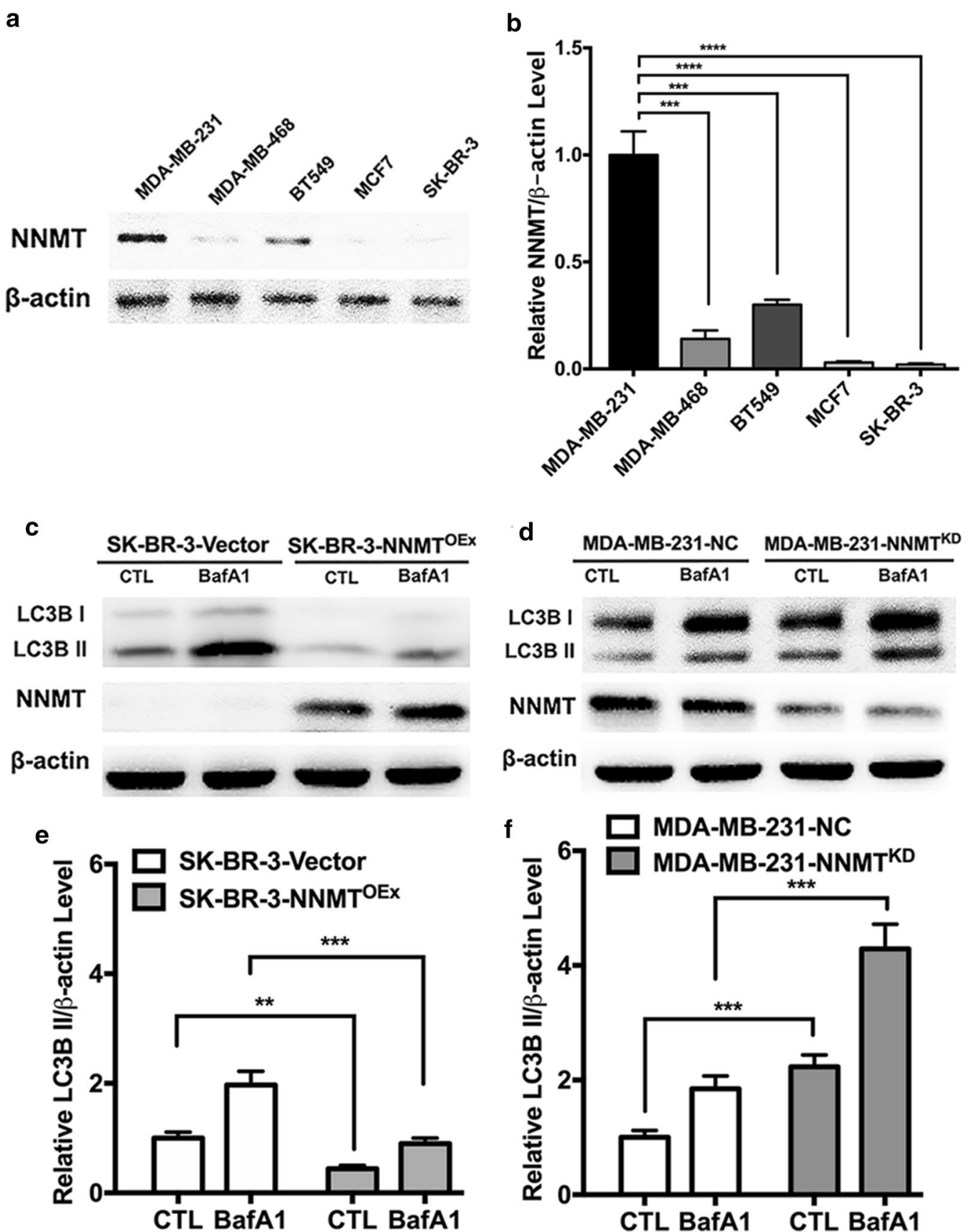

Fig. 1 NNMT inhibited autophagy in breast cancer cell lines. $\mathbf{a}, \mathbf{b}$ The NNMT protein level was determined in the five breast cancer cell lines by Western blotting. $\mathbf{c}, \mathbf{d}$ The LC3B protein level in the two cell models was determined by Western blotting. A representative result from three independent experiments. e, $\mathbf{f}$ The quantification results of $(\mathbf{c})$ and $(\mathbf{d})$, respectively. The protein levels were normalized to $\beta$-actin $\left(^{* *} p<0.01\right.$, ${ }^{* *} p<0.001$ and $\left.{ }^{* * *} p<0.0001\right)$ 
with the highest NNMT expression was infected with the lentivirus of pGCSIL-Pur-shRNA-NNMT to knockdown NNMT or pGCSIL-Pur-shRNA-NC as control (Fig. 1c, d). These two cell models were successfully used to further study NNMT function in on autophagy regulation. After that, we measured autophagic flux in the two model cell lines by examining LC3B II levels with and without bafilomycin A1 (BafA1), a blocker for autophagy substrate degradation. The LC3B II level was reduced by $50 \%$ in SK-BR-3-NNMT ${ }^{\text {OEx }}$ cells compared to that in SK-BR-3-Vector cells with or without BafA1 (Fig. 1c, e), which suggests that NNMT overexpression decreased autophagy flux in breast cancer cells. In contrast, NNMT knockdown significantly increased the LC3B II level in the MDA-MB-231 cell model with or without BafA1 (Fig. 1d, f). This result suggested that NNMT reduces autophagy in breast cancer cells.

\section{NNMT suppresses $\mathrm{H}_{2} \mathrm{O}_{2}$-induced autophagy in breast cancer cells}

To further determine the role of NNMT in autophagy under oxidative stress, we examined autophagy flux in cell models after $\mathrm{H}_{2} \mathrm{O}_{2}$ treatment, which can induce autophagy very well as oxidative stress. The autophagosomes in the two model cell lines after $\mathrm{H}_{2} \mathrm{O}_{2}$ treatment or $\mathrm{H}_{2} \mathrm{O}$ as a control for $8 \mathrm{~h}$ were observed by transmission electron microscopy. The number of autophagosomes was lower in SK-BR-3-NNMT ${ }^{\mathrm{OEx}}$ cells than in SK-BR3 -Vector cells, whereas a higher number of autophagosomes was observed in MDA-MB-231-NNMT ${ }^{\mathrm{KD}}$ cells than in MDA-MB-231-NC cells (Fig. 2a-d), which is consistent with the previous result of the LC3B II assay. With $\mathrm{H}_{2} \mathrm{O}_{2}$ treatment, the numbers of autophagosomes were all increased, indicating that autophagy was induced successfully. The SK-BR-3-NNMT ${ }^{\mathrm{OEx}}$ cells showed a significantly lower number of autophagosomes per cell than SK-BR-3-Vector cells, whereas MDA-MB-231-NNMT ${ }^{\mathrm{KD}}$ cells showed a significantly higher number of autophagosomes than MDA-MB-231-NC cells (Fig. 2a-d). Consistent with the autophagosome results, the LC3B II level was decreased significantly in SK-BR-3 cells after NNMT overexpression and was increased markedly in MDAMB-231 cells after NNMT knockdown both with and without $\mathrm{H}_{2} \mathrm{O}_{2}$ treatment (Fig. 2e-h).

Then, we also monitored autophagosomes by confocal and flow assays using the GFP-LC3 plasmid. In the confocal assay, we observed a lower number of LC3 puncta in SK-BR-3-NNMT ${ }^{\mathrm{OEx}}$ cells than in SK-BR-3-Vector cells with or without $\mathrm{H}_{2} \mathrm{O}_{2}$ treatment (Fig. 3a, b). In contrast, MDA-MB-231-NNMT ${ }^{\mathrm{KD}}$ cells showed the higher number of LC3 puncta than MDA-MB-231-NC cells (Fig. 3c, d). Consistent with the confocal assay results, the mean of GFP-LC3 fluorescence intensity in SK-BR-3-NNMT ${ }^{\mathrm{OEx}}$ cells was significantly lower than that in SK-BR-3-Vector

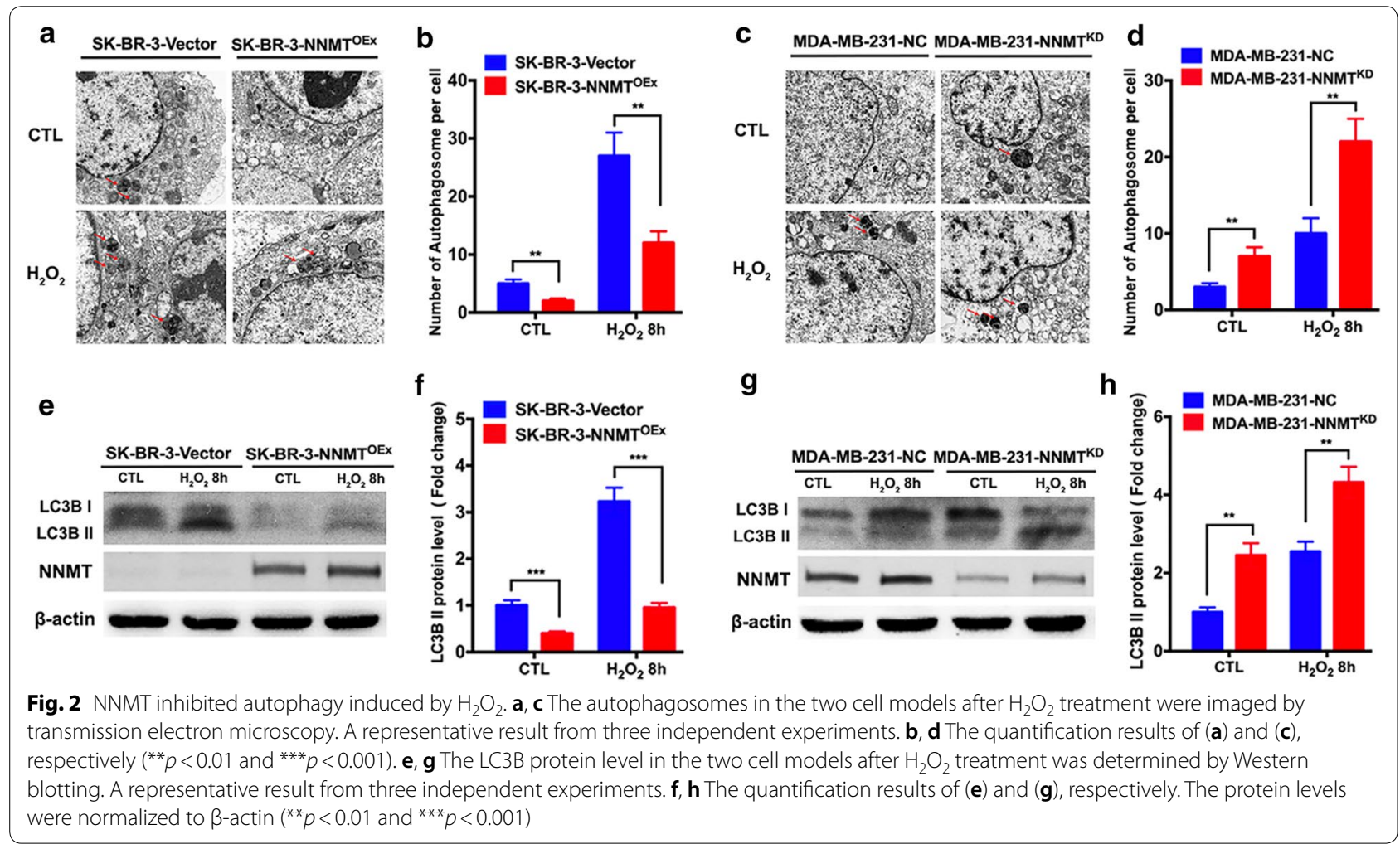



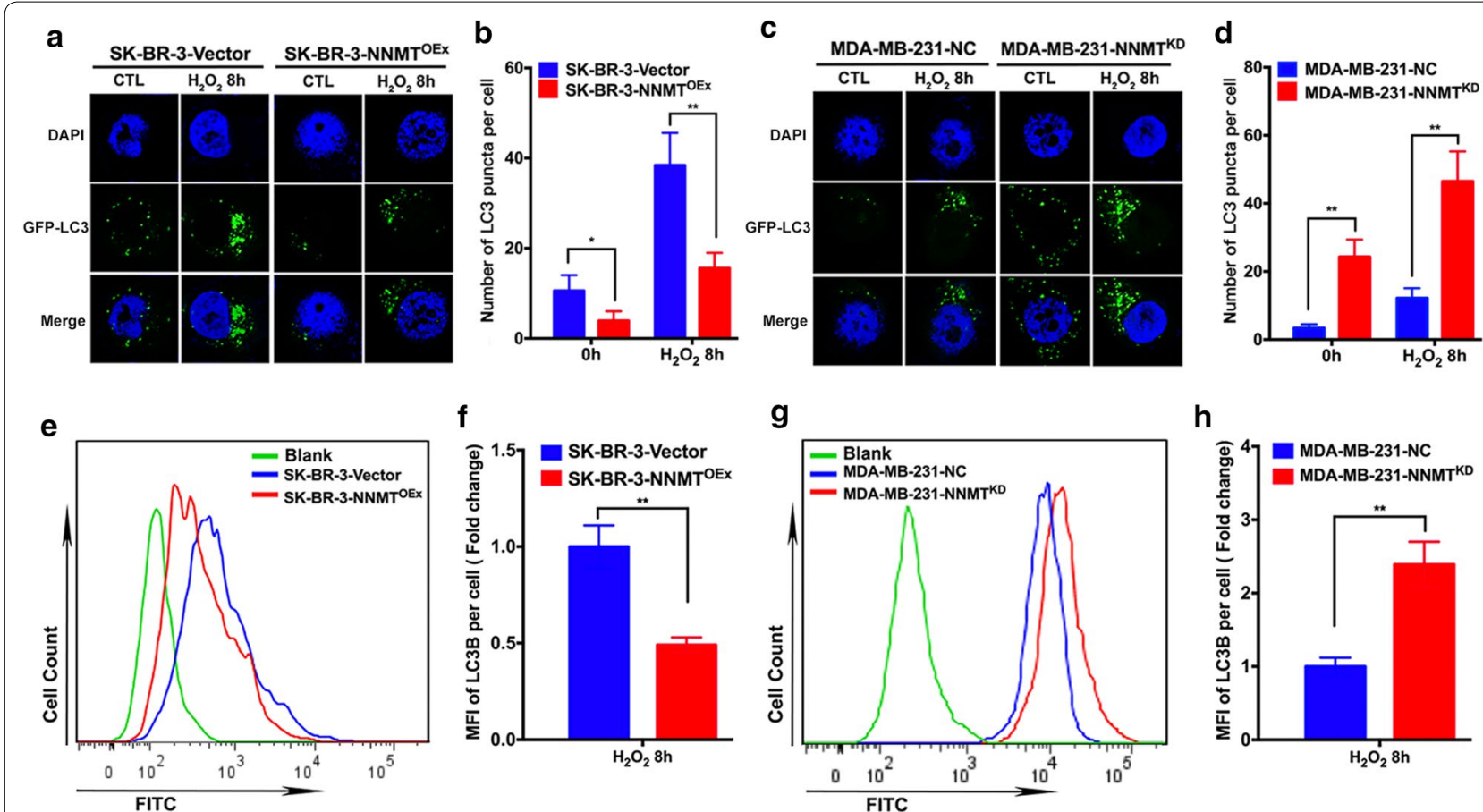

g

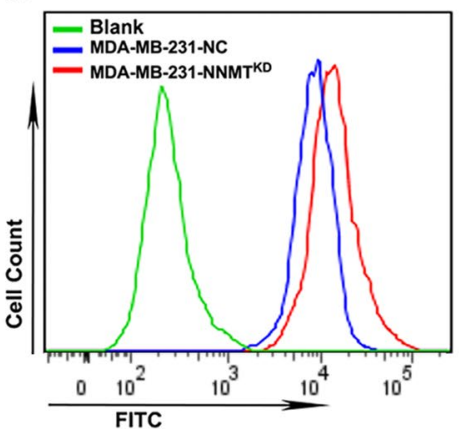

h

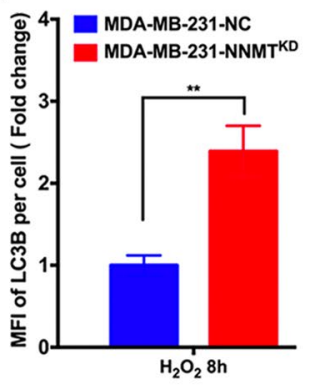

Fig. 3 NNMT decreased LC3 puncta formation induced by $\mathrm{H}_{2} \mathrm{O}_{2}$. a, c The LC3 puncta in the two cell models after $\mathrm{H}_{2} \mathrm{O}_{2}$ treatment were imaged by confocal microscopy. A representative result from three independent experiments. $\mathbf{b}, \mathbf{d}$ The quantification results of (a) and (c), respectively $\left({ }^{* *} p<0.01\right.$ and $\left.{ }^{* * *} p<0.001\right)$. e, $\mathbf{g}$ The fluorescence intensity of LC3B in the two cell models after $\mathrm{H}_{2} \mathrm{O}_{2}$ treatment was assessed by flow cytometry. A representative result from three independent experiments. $\mathbf{f}, \mathbf{h}$ The quantification results of $(\mathbf{e})$ and $(\mathbf{g})$, respectively $\left({ }^{* *} p<0.01\right.$ and $\left.{ }^{* * *} p<0.0001\right)$

cells treated with $\mathrm{H}_{2} \mathrm{O}_{2}$ by flow assay (Fig. 3e, f), whereas MDA-MB-231-NNMT ${ }^{\mathrm{KD}}$ cells showed a more than twofold higher mean GFP-LC3 fluorescence intensity than MDA-MB-231-NC cells (Fig. 3g, h). These results indicated that NNMT negatively regulates autophagy induced by oxidative stress in breast cancer cells.

\section{NNMT decreases the $\mathrm{H}_{2} \mathrm{O}_{2}$-induced autophagy by inhibiting the AMPK-ULK1 pathway in breast cancer cells}

Given that NNMT is inversely correlated with $\mathrm{H}_{2} \mathrm{O}_{2}$-induced autophagy in breast cancer cells, we further explored the potential mechanisms by which NNMT regulates autophagy. First, we assessed the biological function of NNMT in resistance to oxidative stress. After $\mathrm{H}_{2} \mathrm{O}_{2}$ treatment for $8 \mathrm{~h}$, SK-BR-3-NNMT ${ }^{\mathrm{OEx}}$ cells showed the higher cell viability and ATP levels and the lower ROS levels than SK-BR-3-Vector cells. In contrast, MDA-MB-231-NNMT ${ }^{\mathrm{KD}}$ cells showed lower cell viability and ATP levels and higher ROS levels than MDA-MB231-NC cells (Fig. 4). There were all significant differences. These results suggested that NNMT expression decreases the inhibition of cell viability and ATP production and the increases of ROS production, which are related to energy metabolism, resulting in resistance to oxidative stress. Second, we examined the activity of the adenosine monophosphate activated protein kinase (AMPK) pathway after $\mathrm{H}_{2} \mathrm{O}_{2}$ treatment, as it is at the intersection of energy metabolism and autophagy. SKBR-3-NNMT ${ }^{\mathrm{OEx}}$ cells showed a significant lower level of p-AMPK than SK-BR-3-Vector cells both with and without $\mathrm{H}_{2} \mathrm{O}_{2}$ treatment, whereas MDA-MB-231-NNMTKD cells showed a higher level of p-AMPK than MDA-MB231-NC cells (Fig. 5a-d). Furthermore, SK-BR-3-NNM$\mathrm{T}^{\mathrm{OEx}}$ cells showed the lower levels of phosphated-serine/ threonine protein kinase (p-ULK1) (Ser317), which is phosphorylated by AMPK, than SK-BR-3-Vector cells. Similar to the previous result, the LC3B II level was lower in SK-BR-3-NNMT ${ }^{\mathrm{OEx}}$ cells than in SK-BR-3-Vector cells (Fig. 5a, b). In contrast, the p-ULK1 (Ser317) and LC3B II levels were all significantly higher in MDA-MB-231-NN$\mathrm{MT}^{\mathrm{KD}}$ cells than in MDA-MB-231-NC cells (Fig. 5c, d). These results indicated that NNMT decreases the $\mathrm{H}_{2} \mathrm{O}_{2}$-induced autophagy by inhibiting the AMPK-ULK1 pathway in breast cancer cells.

\section{NNMT inhibits the activation of the AMPK-ULK1 pathway via its product $1 \mathrm{MNA}$}

To further clarify the mechanism, we assessed the change in autophagy level in SK-BR-3-Vector and 

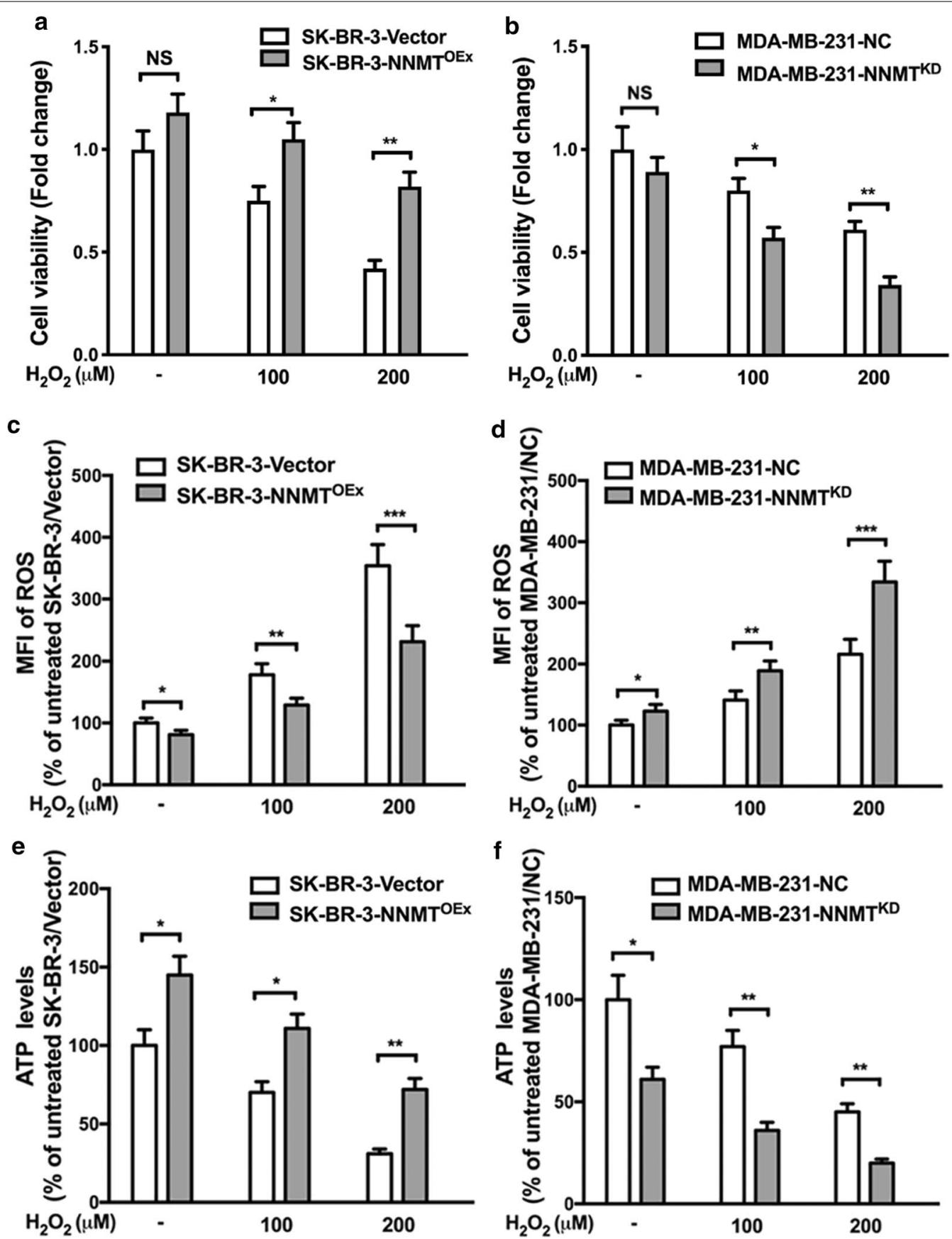

Fig. 4 NNMT decreased the inhibition of cell viability and ATP production and the induction of ROS production induced by $\mathrm{H}_{2} \mathrm{O}_{2}$. $\mathbf{a}, \mathbf{c}$, e The cell viability, intracellular ROS and ATP levels in the SK-BR-3 cell model after $\mathrm{H}_{2} \mathrm{O}_{2}$ treatment were assessed. The data were normalized to the group of SK-BR-3-Vector without $\mathrm{H}_{2} \mathrm{O}_{2}$ treatment, which was defined as 1. b, d, $\mathbf{f}$ The cell viability, intracellular ROS and ATP level in the MDA-MB-231 cell model after $\mathrm{H}_{2} \mathrm{O}_{2}$ treatment was assessed. The data were normalized to those of the MDA-MB-231-NC group without $\mathrm{H}_{2} \mathrm{O}_{2}$ treatment, which was defined as $1\left({ }^{*} p<0.05,{ }^{* *} p<0.01\right.$ and $\left.{ }^{* *} p<0.001\right)$

MDA-MB-231-NNMT ${ }^{\mathrm{KD}}$ cells after 1MNA treatment, which is the product of NNMT. The inhibition of cell viability and ATP production and the increase in ROS production induced by $\mathrm{H}_{2} \mathrm{O}_{2}$ were both reduced in SK-BR-3-Vector cells after treatment with 1MNA $(0.5 \mathrm{mM})$. Additionally, cell viability and ATP production were rescued, and the ROS increase was suppressed by $1 \mathrm{MNA}$ treatment in MDA-MB-231-NNMT ${ }^{\mathrm{KD}}$ cells, 


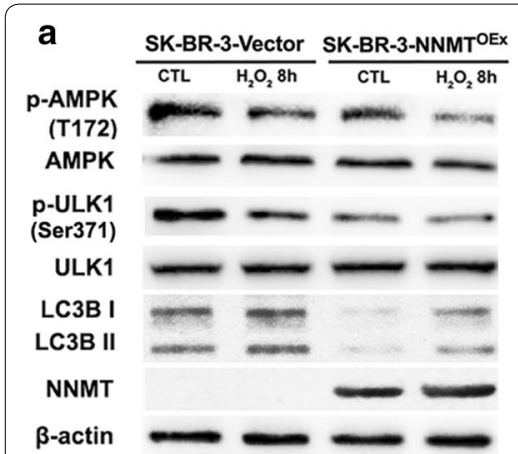

C MDA-MB-231-NC MDA-MB-231-NNMTKD

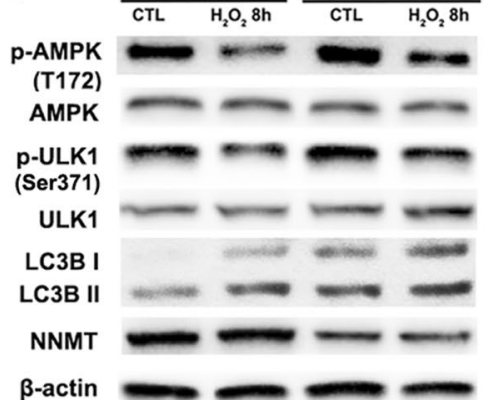

b
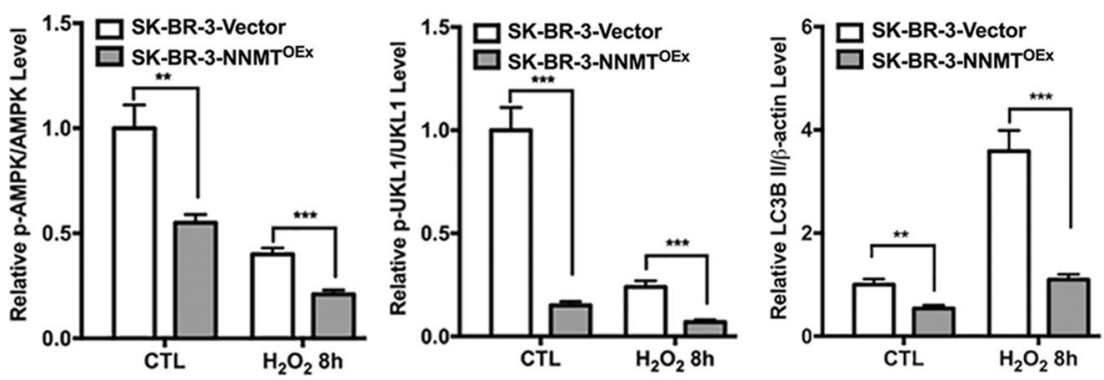

d
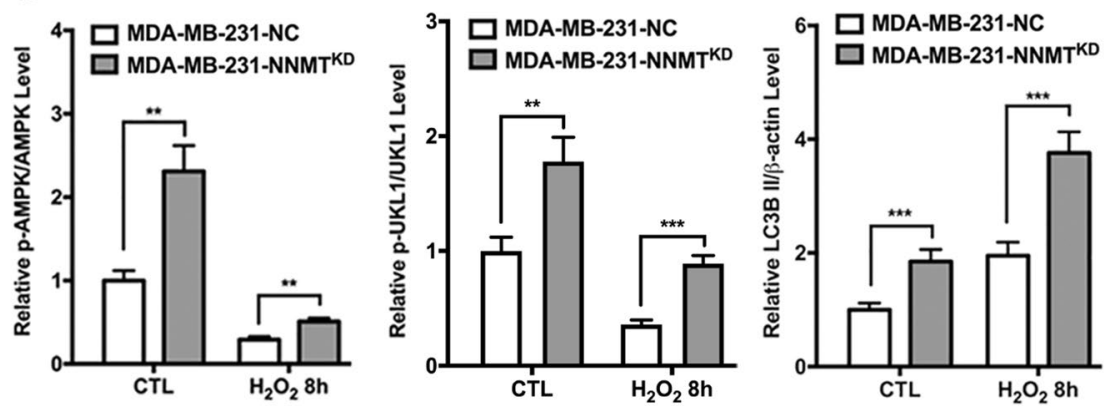

Fig. 5 NNMT inhibited $\mathrm{H}_{2} \mathrm{O}_{2}$-induced autophagy by suppressing the AMPK-ULK1 pathway. a, c The p-AMPK, p-ULK1, AMPK, ULK1 and LC3B protein levels in the two cell models after $\mathrm{H}_{2} \mathrm{O}_{2}$ treatment were determined by Western blotting. A representative result from three independent experiments. b, $\mathbf{d}$ The quantification results of $(\mathbf{a})$ and $(\mathbf{c})$, respectively. The protein levels were normalized to $\beta$-actin $\left(^{* *} p<0.01\right.$ and $\left.{ }^{* * *} p<0.001\right)$

similar to NNMT expression (Fig. 6a-c). These results suggested that 1MNA showed resistance to oxidative stress as well as NNMT expression. Then, we examined the activity of the AMPK-ULK1 pathway and LC3B II levels in SK-BR-3-Vector and MDA-MB-231-NNMT ${ }^{\mathrm{KD}}$ cells after treatment with 1MNA. In both cell line models, 1MNA treatment reduced the activity of the AMPKULK1 pathway and LC3B II levels induced by $\mathrm{H}_{2} \mathrm{O}_{2}$ treatment (Fig. 6d), which indicated that NNMT negatively regulates autophagy under oxidative stress via its product 1MNA.

Together, our results demonstrate that NNMT expression negatively regulates autophagy by inhibiting the activation of the AMPK-ULK1 pathway in breast cancer cells to offer survival advantages to cancer cells under oxidative stress (Fig. 6e). Therefore, NNMT downregulation might be a therapeutic strategy for the combined treatment of breast cancer.

\section{Discussion}

Breast cancer, one of the most prevalent cancers, has the highest mortality rate among cancers in woman worldwide and in China. Therefore, there is an urgent need for new treatment strategies to improve breast cancer treatment.
In recent decades, autophagy has been verified to play a vital role in cancer progression and in response to radiation and chemotherapy, which helps us to shed some light on ways to develop novel strategies for cancer therapy [16]. However, it is generally thought that autophagy has both positive and negative roles in cancer progression, which depends on the particular type of tumour and the stage of cancer progression. In the initial stages, autophagy can inhibit the growth of precancerous cells as a tumour suppressor and decrease the incidence of cancer. During other stages, autophagy may impact the proteome, organelle and metabolism to alter whole cell function. In addition, autophagy also has a survival effect by promoting the adaptation of cancer cells to many stresses, resulting in the resistance to anticancer treatments. Therefore, a comprehensive study of the various contexts involved in the association between autophagy and cancer is beneficial for the novel strategy development of cancer therapy.

Autophagy is a multistep process involving various autophagy-related (ATG) proteins, which are regulated by several upstream signalling pathways, including the AMPK-ULK1 and mTOR pathways [17]. In human cancers, some studies have reported that the ATG genes exhibit low-frequency genomic mutations using largescale genomic analyses, whereas oncogenic events 

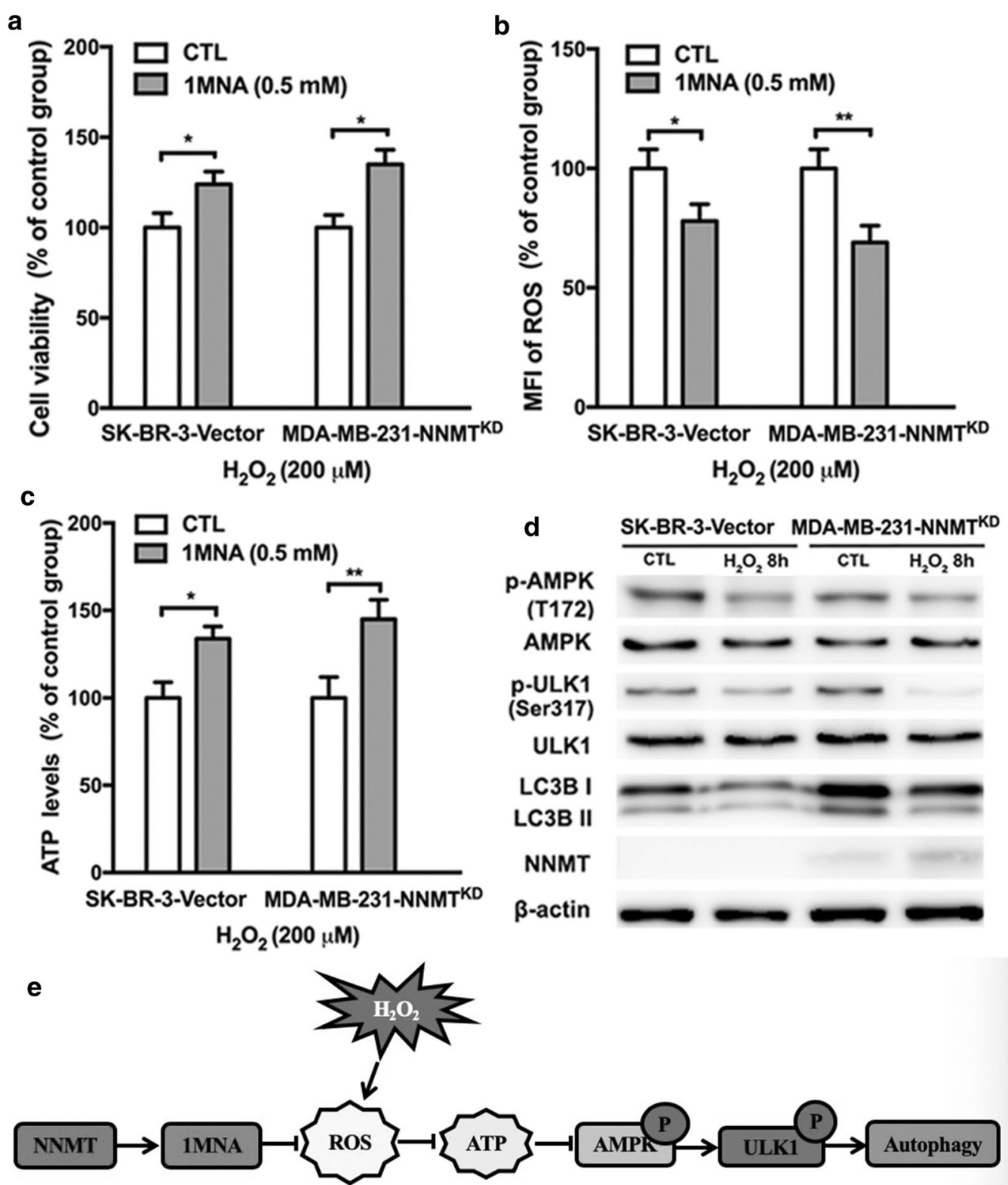

Fig. 6 1MNA inhibited autophagy induced by $\mathrm{H}_{2} \mathrm{O}_{2}$ by reducing the inhibition of cell viability and ATP production and the induction of ROS production. a The cell viability in SK-BR-3-Vector and MDA-MB-231-NNMT KD cells pretreated with and without 1MNA was assessed by MTS after $\mathrm{H}_{2} \mathrm{O}_{2}$ treatment. b The intracellular ROS level in SK-BR-3-Vector and MDA-MB-231-NNMT ${ }^{\mathrm{KD}}$ cells pretreated with and without $1 \mathrm{MNA}$ was assessed by flow cytometry after $\mathrm{H}_{2} \mathrm{O}_{2}$ treatment. c The intracellular ATP level in SK-BR-3-Vector and MDA-MB-231-NNMT ${ }^{\mathrm{KD}}$ cells pretreated with and without 1 MNA was assessed by an ATP kit after $\mathrm{H}_{2} \mathrm{O}_{2}$ treatment. The SK-BR-3-Vector and MDA-MB-231-NNMT ${ }^{\mathrm{KD}}$ groups without 1 MNA treatment were considered as the control groups, and their values were normalized to $100 \%$. $\left({ }^{*} p<0.05\right.$, and $\left.{ }^{* *} p<0.01\right)$ d The p-AMPK, $p-U L K 1, A M P K$, ULK1 and LC3B protein levels in SK-BR-3-Vector and MDA-MB-231-NNMT ${ }^{\mathrm{KD}}$ cells pretreated with and without 1MNA were determined by Western blotting after $\mathrm{H}_{2} \mathrm{O}_{2}$ treatment. e Schematic model of this study

regulate the cancer-related signalling pathways and consequently lead to abnormal autophagy [18]. One of the earliest discoveries was that $\mathrm{p} 53$, a frequently inactivated or mutated transcription factor, can trigger autophagy in cancer cells [19]. The AKT signalling pathway, which promotes cancer cell proliferation, can suppress the TSC1/2 complex, resulting in mTOR1 activation and autophagy inhibition.

NNMT was discovered as a cancer-related protein in recent years. As a methyltransferase, NNMT transfers a methyl group from SAM to NAM to produce 1MNA and $\mathrm{SAH}$, which impacts the global methylation status in cells 
[20]. NNMT has been recently reported to participate in the development and progression of various carcinomas and regulate cancer metabolism [12]. In breast cancer, we have found that NNMT and its product 1MNA can inhibit the mitochondrion-mediated apoptosis through decreasing intracellular ROS, which enhances the resistance to chemotherapy by SIRT1 protein stabilization [11, 15]. Considering that the NNMT gene was predicted to be an autophagy regulator by genome-wide analysis [21], we investigated the role of NNMT in the autophagy of breast cancer cells.

The regulation of oxidative stress is pivotal in both autophagy and tumorigenesis, since ROS can modulate many signalling pathways involved in autophagy and tumorigenesis through direct or indirect ways [22, 23]. As a second messenger, low to moderate levels of ROS can regulate the activation or expression of multiple signalling proteins such as mitogen-activated protein kinase (MAPK), AMPK, protein kinase B (AKT), extracellular signal-regulated kinase (ERK), c-Jun $\mathrm{N}$-terminal kinase (JNK) [24-29], all of which are involved in cancer cell survival, proliferation and stress response. In a previous study, we reported that NNMT expression increases its product 1MNA level in cancer cells, which decreases intracellular ROS levels to inhibit mitochondria-mediated apoptosis in breast cancer cells and to suppress 5 -FU-induced apoptosis in colorectal cancer cells $[15,30]$. In this study, even though NNMT inhibited autophagy under normal culture conditions, the NNMT effect was more distinct under oxidative stress. Furthermore, we found that NNMT and its product 1MNA suppress the increase in ROS induced by $\mathrm{H}_{2} \mathrm{O}_{2}$, which may protect mitochondria and cell viability against oxidative stress and inhibit the activation of AMPK induced by oxidative stress in breast cancer cells. AMPK can sense energy changes in cells and maintain cellular energy homeostasis by regulating energy-related metabolism. When the ratio of ATP:ADP in cells is low, AMPK can be activated to directly increase the phosphorylation level of ULK1 at Ser317 and Ser777, which promotes autophagy. Therefore, the AMPK-ULK1 pathway is regarded as the master upstream regulated signalling pathway of autophagy, especially under oxidative and energy stress [31]. In our study, we found that NNMT and its product 1MNA inhibit the activation of the AMPK-ULK1 pathway by suppressing ROS production in breast cancer cells after $\mathrm{H}_{2} \mathrm{O}_{2}$ treatment.

Combined with our results, we hypothesized that NNMT and its product 1MNA negatively regulate autophagy by suppressing the increase in ROS induced by oxidative stress in breast cancer cells. Consistent with our findings, Skin et al. found that NNMT decreases the PP2A methylation level by methyl group transfer from SAM to NAM to inhibit autophagy in liver cancer cells [32]. However, Schmeisser et al. recently reported that NNMT induces autophagy in some animal models by reducing of its substrate SAM [33]. NNMT regulates its substrate SAM and NAM and its product 1MNA levels, which are involved in many important biological processes, including cellular response to stress and cellular energy production. The three molecules maintain a different balance in different types of cells and each of them performs its own function, which may result in the complex function of NNMT in different types of cells. Thus, although NNMT plays a role in regulating autophagy, the detailed mechanisms in different cells require further study.

In addition, cell survival and death are particularly complex processes, and autophagy and apoptosis both play significant roles, between which there is a close association. Our previous and current studies have shown that NNMT plays a role both in regulating apoptosis and autophagy in breast cancer. However, the detailed mechanism by which NNMT regulates the balance between apoptosis and autophagy in breast cancer cells needs further study.

\section{Conclusions}

In this study, we first found that NNMT is involved in autophagy regulation in breast cancer cells. Furthermore, our results suggest that NNMT expression decreases the inhibition of cell viability and ATP production and the increase in ROS production induced by oxidative stress and demonstrated that NNMT and its product 1MNA negatively regulate autophagy to protect breast cancer cells against oxidative stress by suppressing the activation of the AMPK-ULK1 pathway, these findings may provide a novel strategy of targeting NNMT for the combined treatment of breast cancer.

\section{Abbreviations}

NNMT: Nicotinamide N-methyltransferase; ROS: Reactive oxygen species; SAM: S-adenosyl-L-methionine; NAM: Nicotinamide; 1MNA: 1-methylnicotinamide; SAH: S-adenosylhomocysteine; ATCC: American type culture collection; BafA1: Bafilomycin A1; ATG: Autophagy-related; AMPK: Adenosine monophosphate activated protein kinase; MAPK: Mitogen-activated protein kinase; ERK: Extracellular signal-regulated kinase; ULK1: Serine/threonine protein kinase; AKT: Protein kinase B; JNK: c-Jun N-terminal kinase.

\section{Acknowledgements}

None.

\section{Authors' contributions}

JZ conceived and designed the experiments; $\mathrm{HY}$ designed and performed the experiments and wrote the paper; $X Z$ designed and performed the experiments; $X C H$, JZ, JY and GLL performed the experiments; $Y Z W$ analysed the data; $X Y X$ critically reviewed and edited the manuscript. All authors read and approved the final manuscript. 


\section{Funding}

This work was supported by grants from National Natural Science Foundation of China (No. 81271914), Key Research and Development Program of Zhejiang Province (No. 2019C03021), Key Traditional Chinese Medicine Program of Zhejiang Province (No. 2018ZZ016), Natural Science Foundation of Zhejiang Province (Nos. LGC19H200005 and LY12H16025), and Health Bureau Foundation of Zhejiang Province (Nos. 2018KY110, 2018KY482, 2015RCA016 and 2015KYA143).

\section{Availability of data and materials}

The datasets generated during the current study are available from the corresponding author on reasonable request.

\section{Ethics approval and consent to participate}

Not applicable.

\section{Consent for publication}

Not applicable.

\section{Competing interests}

The authors declare that they have no competing interests.

\begin{abstract}
Author details
${ }^{1}$ Department of Clinical Laboratory, Sir Run Run Shaw Hospital, Zhejiang University School of Medicine, 3 East Qingchun Road, Hangzhou 310016, Zhejiang, People's Republic of China. ${ }^{2}$ Key Laboratory of Biotherapy of Zhejiang Province, 3 East Qingchun Road, Hangzhou 310016, Zhejiang, People's Republic of China. ${ }^{3}$ Department of Clinical Laboratory, Xiasha campus, Sir Run Run Shaw Hospital, Zhejiang University School of Medicine, 368 Xiasha Road, Hangzhou 310018, Zhejiang, People's Republic of China.
\end{abstract}

Received: 19 July 2019 Accepted: 18 May 2020

Published online: 24 May 2020

\section{References}

1. Ravikumar B, Sarkar S, Davies JE, Futter M, Garcia-Arencibia M, GreenThompson ZW, et al. Regulation of mammalian autophagy in physiology and pathophysiology. Physiol Rev. 2010;90:1383-435.

2. Green DR, Levine B. To be or not to be? How selective autophagy and cell death govern cell fate. Cell. 2014;157:65-75.

3. Mizushima N, Komatsu M. Autophagy: renovation of cells and tissues. Cell. 2011;147:728-41.

4. Chen GQ, Gong RH, Yang DJ, Zhang G, Lu AP, Yan SC, et al. Halofuginone dually regulates autophagic flux through nutrient-sensing pathways in colorectal cancer. Cell Death Dis. 2017;8:e2789.

5. Cheng Y, Ren X, Hait WN, Yang JM. Therapeutic targeting of autophagy in disease: biology and pharmacology. Pharmacol Rev. 2013;65:1 162-97.

6. Kraus D, Yang Q, Kong D, Banks AS, Zhang L, Rodgers JT, et al. Nicotinamide N-methyltransferase knockdown protects against diet-induced obesity. Nature. 2014;508:258-62.

7. Jang JS, Cho HY, Lee YJ, Ha WS, Kim HW. The differential proteome profile of stomach cancer: identification of the biomarker candidates. Oncol Res. 2004;14:491-9.

8. Roessler M, Rollinger W, Palme S, Hagmann ML, Berndt P, Engel AM, et al. Identification of nicotinamide $\mathrm{N}$-methyltransferase as a novel serum tumor marker for colorectal cancer. Clin Cancer Res. 2005;11:6550-7.

9. Kim J, Hong SJ, Lim EK, Yu YS, Kim SW, Roh JH, et al. Expression of nicotinamide $\mathrm{N}$-methyltransferase in hepatocellular carcinoma is associated with poor prognosis. J Exp Clin Cancer Res. 2009;28:20.

10. Chen C, Wang X, Huang X, Yong H, Shen J, Tang Q, et al. Nicotinamide $\mathrm{N}$-methyltransferase: a potential biomarker for worse prognosis in gastric carcinoma. Am J Cancer Res. 2016;6:649-63.

11. Wang Y, Zeng J, Wu W, Xie S, Yu H, Li G, et al. Nicotinamide N-methyltransferase enhances chemoresistance in breast cancer through SIRT1 protein stabilization. Breast Cancer Res. 2019;21:64.

12. Hong S, Moreno-Navarrete JM, Wei X, Kikukawa Y, Tzameli I, Prasad D, et al. Nicotinamide $\mathrm{N}$-methyltransferase regulates hepatic nutrient metabolism through Sirt1 protein stabilization. Nat Med. 2015;21:887-94.

13. Eckert MA, Coscia F, Chryplewicz A, Chang JW, Hernandez KM, Pan S, et al. Proteomics reveals NNMT as a master metabolic regulator of cancerassociated fibroblasts. Nature. 2019;569:723-8.
14. Ulanovskaya OA, Zuhl AM, Cravatt BF. NNMT promotes epigenetic remodeling in cancer by creating a metabolic methylation sink. Nat Chem Biol. 2013;9:300-6.

15. Zhang J, Wang Y, Li G, Yu H, Xie X. Down-regulation of nicotinamide $\mathrm{N}$-methyltransferase induces apoptosis in human breast cancer cells via the mitochondria-mediated pathway. PLoS ONE. 2014;9:e89202.

16. Zhang W, Wan X, Liu Z, Xiao L, Huang H, Liu X. The emerging role of oxidative stress in regulating autophagy: applications of cancer therapy. Cell Mol Biol. 2017;63:67-76.

17. Galluzzi L, Pietrocola F, Levine B, Kroemer G. Metabolic control of autophagy. Cell. 2014;159:1263-76.

18. Lebovitz CB, Robertson AG, Goya R, Jones SJ, Morin RD, Marra MA, et al. Cross-cancer profiling of molecular alterations within the human autophagy interaction network. Autophagy. 2015;11:1668-87.

19. Tasdemir E, Maiuri MC, Galluzzi L, Vitale I, Djavaheri-Mergny M, D'Amelio $\mathrm{M}$, et al. Regulation of autophagy by cytoplasmic p53. Nat Cell Biol. 2008;10:676-87.

20. Aksoy S, Szumlanski CL, Weinshilboum RM. Human liver nicotinamide $\mathrm{N}$-methyltransferase. cDNA cloning, expression, and biochemical characterization. J Biol Chem. 1994;269:14835-40.

21. Lipinski MM, Hoffman G, Ng A, Zhou W, Py BF, Hsu E, et al. A genomewide siRNA screen reveals multiple mTORC1 independent signaling pathways regulating autophagy under normal nutritional conditions. Dev Cell. 2010;18:1041-52.

22. Gorrini C, Harris IS, MakTW. Modulation of oxidative stress as an anticancer strategy. Nat Rev Drug Discov. 2013;12:931-47.

23. Lin WJ, Kuang HY. Oxidative stress induces autophagy in response to multiple noxious stimuli in retinal ganglion cells. Autophagy. 2014;10:1692-701.

24. Ito K, Hirao A, Arai F, Takubo K, Matsuoka S, Miyamoto K, et al. Reactive oxygen species act through p38 MAPK to limit the lifespan of hematopoietic stem cells. Nat Med. 2006;12:446-51.

25. Tsugawa H, Suzuki H, Saya H, Hatakeyama M, Hirayama T, Hirata K, et al. Reactive oxygen species-induced autophagic degradation of Helicobacter pylori CagA is specifically suppressed in cancer stem-like cells. Cell Host Microbe. 2012;12:764-77.

26. Kano G, Almanan M, Bochner BS, Zimmermann N. Mechanism of Siglec8-mediated cell death in IL-5-activated eosinophils: role for reactive oxygen species-enhanced MEKJERK activation. J Allergy Clin Immunol. 2013;132:437-45

27. Tang HW, Liao HM, Peng WH, Lin HR, Chen CH, Chen GC. Atg9 interacts with dTRAF2/TRAF6 to regulate oxidative stress-induced JNK activation and autophagy induction. Dev Cell. 2013;27:489-503.

28. Zhang Y, Choksi S, Chen K, Pobezinskaya Y, Linnoila I, Liu ZG. ROS play a critical role in the differentiation of alternatively activated macrophages and the occurrence of tumor-associated macrophages. Cell Res. 2013;23:898-914.

29. Hart PC, Mao M, de Abreu AL, Ansenberger-Fricano K, Ekoue DN, Ganini $D$, et al. MnSOD upregulation sustains the Warburg effect via mitochondrial ROS and AMPK-dependent signalling in cancer. Nat Commun. 2015;6:6053.

30. Xie X, Liu H, Wang Y, Zhou Y, Yu H, Li G, et al. Nicotinamide N-methyltransferase enhances resistance to 5-fluorouracil in colorectal cancer cells through inhibition of the ASK1-p38 MAPK pathway. Oncotarget. 2016;7:45837-48.

31. Mercer TJ, Gubas A, Tooze SA. A molecular perspective of mammalian autophagosome biogenesis. J Biol Chem. 2018;293:5386-95.

32. Shin JH, Park CW, Yoon G, Hong SM, Choi KY. NNMT depletion contributes to liver cancer cell survival by enhancing autophagy under nutrient starvation. Oncogenesis. 2018;7:58.

33. Schmeisser K, Parker JA. Nicotinamide-N-methyltransferase controls behavior, neurodegeneration and lifespan by regulating neuronal autophagy. PLoS Genet. 2018;14:e1007561.

\section{Publisher's Note}

Springer Nature remains neutral with regard to jurisdictional claims in published maps and institutional affiliations. 